https://doi.org/10.22519/22157360.705

\title{
El poder, la política y estado en el absolutismo y el
}

\author{
renacimiento
}

\section{Power, politics and state and the absolutism and renaissance}

\author{
Omaira Bernal Payares ${ }^{1}$
}

\section{Resumen}

En el estudio del poder se da una variedad de eventos a través de las diferentes épocas que ha pasado la humanidad, en el absolutismo se presentaron a través de monarquías nacionales y absolutas, abarcando los siglos XVI al XVIII y define el nacimiento de la era moderna, negando el feudalismo medieval para dar paso a un estado de corte absoluto.

Este artículo tiene como objetivo caracterizar la manera como se presentó las ideas de poder, la política y el Estado en los tiempos del absolutismo, presentando los aportes de los principales teóricos de esos tiempos.

Palabras clave: Poder; política; estado; absolutismo; renacimiento; reforma.

\begin{abstract}
A variety of events through different times happened humanity, absolutism is given in the study of power were presented by national and absolute monarchies, encompassing the

Docente Investigadora Corporación Universitaria Rafael Núñez omaira.bernal@curnvirtual.edu.co CÓDIGO JEL: Z19

\footnotetext{
${ }^{1}$ Magister en Administración y Administradora de la Salud Candidata a doctora en Ciencias Sociales énfasis Gerencia Universidad de Zulia AGLALA ISNN 2215-7360
}

2014; 5 (1): 168-183
\end{abstract}


Fecha de recepción: Julio de 2014 / Fecha de aceptación en forma revisada: Octubre 2014

sixteenth to the eighteenth and define the birth of the modern era denying medieval feudalism giving way to a state of absolute cut.

This paper aims to characterize how the ideas presented power, politics and the state in times of absolutism, featuring contributions from leading theorists of the time.

Keywords: Power; politic; state; absolutism; renaissance; reform.

\section{Introducción}

El absolutismo durante la era medieval del renacimiento comprende los siglos XV al XVIII, se le conoce como el comienzo de la edad moderna debido a que en esos tiempos se presentaron sucesos históricos que dieron nacimiento a grandes cambios, se despliega el estado absolutista y se da el renacimiento de la humanidad en las artes, la ciencia y la filosofía.

En el siglo XVI se dio una reforma que se presentó como un movimiento dentro de la cristiandad occidental para depurar la iglesia de las arbitrariedades medievales y devolver las enseñanzas y prácticas que, según expresaban los Reformadores, tenían relación con la Biblia y con la forma de iglesia en el Nuevo Testamento. Esto produjo un rompimiento entre la Iglesia Católica Romana y los reformadores, cuyas formas de pensar y de practicar su fe se llamaron protestantes.

El humanismo renacentista estuvo enfocado con la renovación de la persona, la valoración del mundo del ser humano, así como su génesis y libertad para tomar decisiones y dar opinión. 
Este artículo tiene como objetivo caracterizar la manera como se presentó las ideas de poder, la política y el Estado en los tiempos del absolutismo, presentando los aportes de los principales teóricos de esos tiempos.

\section{La política en la era absolutista}

La extensa crisis económica que las sociedades europeas tuvieron durante los siglos XIV y XV manifestó los problemas y límites de la manera feudal de producción. Durante el siglo XVI apareció en Occidente el Estado absolutista. Las monarquías centralizadas de Francia, Inglaterra y España representaron la ruptura de manera decisiva con las sociedades medioevales. Este fue también el inicio de la edad moderna, se dan grandes cambios; todo esto aparejada con el renacimiento de las artes, la ciencia y la filosofía.

Ávila y Guillezeau en el año 2009 expresan que la complejidad y la multidimensionalidad de la filosofía política se dan porque sus teorías, desde el ámbito político, vienen de la formación de las monarquías nacionales y absolutas, además de los ideales de la reforma.

El absolutismo se da con fuerza debido a la rápida expansión de las actividades, los recursos económicos y el poder interno de los reyes dentro de tales estados absolutistas, debido a ello, el poder se concentra en el rey. A su vez, se da el reinicio de una filosofía política que rompe con la filosofía medioeval, que estuvo fundada por lo místico religioso.

El platonismo y el Aristotelismo surgen de manera paralela durante esta época. Estos plantean recuperar la doctrina auténtica de aquellos, el cual fue deformada durante el Medioevo. A través del aristotelismo, se da la posibilidad de encontrar un orden racional y necesario en el mundo y que excluye a la divinidad.

AGLALA ISNN 2215-7360

2014; 5 (1): 168-183 
Como doctrina política el absolutismo afirma que el rey es el único que debe de crear leyes y ejercer el poder sin ningún obstáculo debido que tal poder le es dado por Dios. Esta doctrina se presentó en Inglaterra, por medio de la dinastía de los Tudor, en Francia, a través de Enrique XIV y en España, con Carlos I y su hijo Felipe II. En estas monarquías la autoridad se sustentó por medio de las tradiciones.

Perry (1987), expresa que durante el siglo XVI, tras la llegada del absolutismo, esta no fue un proceso delicado para las clases dominantes, sino que se presentaron rupturas y conflictos fueres en extremo en el interior de la aristocracia feudal. Además, el absolutismo fue un aparato potencializado de dominación feudal, que procuró mantener al campesinado en su posición social tradicional, por lo cual el Estado absolutista se constituyó como una nueva forma de dominio de la nobleza.

Maquiavelo escribe en el año 1513 la obra El Príncipe, dentro del cual se analizan los diferentes tipos de gobierno de tal época, traza los rasgos del gobernante y los métodos que debe de hacer para mantenerse en el poder. Además, expresa que a través del poder se debe de hacer a un lado los aspectos éticos o morales a favor de los intereses del Estado y la eficacia.

Se presentaron otros teóricos que apoyaron el estado absolutista, los cuales fueron Jean Bodin, que publicó en el año 1576 Los Seis Libros de la República. Ahora, Fazzio (2006), al hablar de Bodin, dice que este personaje intentó dar un fundamento sólido al poder real por medio de una doctrina sobre la soberanía. Además, señala que un Estado existe cuando los ciudadanos están sometidos a la ley de un soberano. Tal soberanía da poder perpetuo, ilimitado, no se delega, no prescribe ni es limitado por las leyes, debido a que el mismo rey es la fuente de las leyes. Ante esto, el soberano puede decretar la paz o la guerra, dar su consentimiento para los que ejercerán las funciones del estado, su veredicto es inapelable, puede conceder gracia, AGLALA ISNN 2215-7360

2014; 5 (1): 168-183 
acuñar monedas y de manera impositiva dictar los impuestos. Sin embargo, estos soberanos deben de estar sujetos a las leyes de Dios.

Otro teórico fue Tomas Hobes (citado en Ávila y Gillezeau, 2009), el cual racionaliza al Estado absolutista y le da su justificación de existencia. Expresó que las naciones experimentarán prosperidad por medio de la monarquía debido a que obedecen al monarca. Además, afirmó que para que en las sociedades se pueda convivir es necesario ceder parte de los derechos del ciudadano al Estado y pues, era el monarca el que concentraba todos los derechos que fueron cedidos por los individuos.

Mientras tanto, Jacques Bossuet, (citado en Ávila y Gillezeau, 2009), en su obra Política sacada de las Sagradas Escrituras, funda su reflexión en el texto sagrado bíblico y del derecho romano. Ante ello, afirmó que el trono es del mismo Dios representado por un hombre, por lo tanto era sagrada y absoluta.

El poder del monarca se presentaba de manera vitalicia, y pues al fallecer, el poder pasaba a manos del hijo. Tal rey no se ponía bajo ningún control, ni tampoco hacía partícipe su soberanía con ninguna otra persona.

Las monarquías absolutas de occidente, según Perry (1987), se basaron en un cualificado estrato de legistas que suplían de personal a la administración de los Estados. Estos eran los letrado en España, los maîtres des requetés en Francia, y los doctores en Alemania. Estos fueron celosos defensores del centralismo monárquico.

En el ejercicio del poder, el monarca tenía un Consejo de Estado, el cual era designado por el propio rey, además de otras áreas especializadas como eran los consejos de finanzas, de justicia, de guerra y otros.

AGLALA ISNN 2215-7360

2014; 5 (1): 168-183 
La forma como se integró la nobleza feudal en el Estado absolutista fue a través de la adquisición de cargos, los cuales son comprados por los burgueses y particulares. Esto llevó a que se formara la clase de los empleados. Romano y Terenti (1986), en su aporte a este punto, expresa que el funcionario que forma parte del Estado se adueña del poder y empieza a edificar una clase en sí mismo, presentándose una corrupción burocrática.

El estrato social se dividió en tres estamentos: la nobleza, el clero y el tercer estado. Estos presentaron desigualdades a nivel jurídico y fiscal. La nobleza y el clero se consideraban privilegiados. Los primeros eran cerrados y se accedía por medio del nacimiento, aunque muchos burgueses con riquezas compraban títulos nobiliarios. Los segundos, tuvieron gran influencia cultural y política, tuvieron propiedades y privilegios fiscales. Se presentaron por medio del alto clero y el bajo clero. En cambio, el tercer estado, se consideró en no privilegiado, donde estuvo la mayoría de la población. Estos no formaban parte de la nobleza y del clero, sino lo integraron los burgueses, los campesinos, los artesanos y los trabajadores urbanos. La mayoría no poseían tierras o tenían recursos escasos y dependían de los señores.

Nuevamente con Perry (1987), la diplomacia se constituyó uno de las grandes invenciones institucionales de aquellos tiempos, lo cual dio inicio al Estado Renacentista. Tal diplomacia comenzó en Italia en el siglo XV y fue tomada como ejemplo por España, Francia, Inglaterra, Alemania y toda Europa durante el siglo XVI. Se constituyeron embajadas en otros países y se nombraron a cancilleres que se encargaron de las relaciones exteriores.

AGLALA ISNN 2215-7360

2014; 5 (1): 168-183 


\section{El mercantilismo y los estados absolutistas}

El poder del estado absolutista se puede observar en su reflejo en la economía, el mercantilismo es su política económica por lo que se empezó a implementarse a mediados del siglo XV y su declinación se dio a mediados del siglo XVIII.

El mercantilismo niega la forma como se llevaba la economía en el medioevo, por lo que busca sentar las columnas para que se manifieste el desarrollo mercantil y manufacturero. Esto hizo que naciera una nueva clase social que se formó en las ciudades. Estos eran individuos que, aunque no eran letrados ni cultos, tenían actividades económicas como fueron los banqueros, funcionarios y comerciantes que se centraron en enriquecer a la nación.

Fabal (1973) expone las principales medidas en materia económica que se impusieron en los estados de corte absolutista en medio del mercantilismo: a) Se aplicó un proteccionismo arancelario en el aspecto aduanero, b) en el aspecto comercial, se promovió las exportaciones y se redujo las importaciones para generar una balanza de pagos favorable, c) por el coloniaje en otros territorios garantizaba nuevas áreas para la exportación a la metrópoli, y d) la explotación de minas por el estado.

A los mercantilistas no les interesaban las teorías de la producción y/o el consumo, y lo que a ellos les interesaba era el aumento del poder del estado, por no en oposición al sector privado, sino a otros estados, la iglesia y los señores feudales. El mercantilismo, entonces, estuvo enfocado en dirección a la geopolítica y la economía y, dadas las bajas tasas de crecimiento económico, se suponía que la riqueza y el poder mundial eran tener mayor cantidad de bienes, por lo que un aumento de los tales traería un incremento relativo para un país; sin embargo, muchas veces ese crecimiento era a expensas de los demás países; con frecuencia, a través de guerras.

AGLALA ISNN 2215-7360

2014; 5 (1): 168-183 


\section{El humanismo en el renacimiento}

Durante el periodo absolutista se da un desarrollo creativo que cambia la forma de vida en las artes, la filosofía y la ciencia. En el siglo XVI se presenta el renacimiento, en el siglo XVII se le llamó El Barroco, debido a que utilizaba el exceso de énfasis y ornamentación en las artes y el siglo XVIII se le denominó La Ilustración, que se presentó como una época donde se resaltaba la racionalidad clara y sobria, el poder de la razón del ser humano, de la ciencia y el respeto hacia la humanidad.

El centro donde se originó el renacimiento se da en Florencia (Italia) para luego extenderse en toda Europa occidental. El eje por el cual se basa fue el humanismo, negando la tradición del medioevo representada por la escolástica, fue que el hombre es el centro de las artes y del pensamiento occidental.

Ahora, el siglo XV se da el surgimiento de la edad moderna, pero hay que tener presente que no fue un rompimiento a la vez de la edad media, sino que se dio de manera paulatina la transformación.

Por otro lado, durante el siglo XV sucedieron tres grandes acontecimientos que dieron como resultado cambios en Europa que fueron: el uso de la pólvora en las armas, la brújula y la imprenta.

Los humanistas alegaban que el ser humano es el centro y medida de todas las cosas, consideraban un orgullo las realizaciones de la humanidad con libertad y de hecho superior a todas las demás criaturas. Esto contradice el pensamiento medieval que consideraba importante la vida espiritual o ultraterrenal.

Echandi en el año 2007 expresaba que los humanistas buscaron dar respuesta a las interrogantes que se presentaron en esos tiempos y por ello buscaron en el cristianismo y en la AGLALA ISNN 2215-7360

2014; 5 (1): 168-183 
filosofía grecolatina, trayendo como resultado un intelectualismo que se caracterizó por la supremacía del ser humano sobre la naturaleza y el rechazo de las creencias impuestas por la religión de la era medieval. Además, se buscaba que el hombre tuviera un espíritu crítico y que confiara en sus propias posibilidades que le fueron vetados en la edad media.

\section{Poder y política de los principales representantes del absolutismo}

Hobbes (citado en Ávila y Gillezeau, 2009), reforzó la teoría del poder y la política, en su concepción de la ley, de la justicia, del Estado, del ser humano. Expresa que la inclinación perpetua de la humanidad es el afán de poder que termina con la muerte. Además, en la naturaleza humana se encuentra la competencia, la desconfianza y la gloria. Debido a ello, mientras viva el individuo sin un poder que de temor a todos, se encuentra en una situación de guerra, el cual es una guerra de todos contra todos. Sin embargo, se puede superar esa condición por medio de sus pasiones, que da a entender la búsqueda de la paz y el privilegio del otro y, por la razón, debido a que sin seguridad y duración, los bienes y privilegios deseados no tienen sentido porque no se pueden disfrutar. La razón sugiere normas adecuadas de paz y que mediante el consenso se puede llegar. A esto Hobbes lo llama Leyes de la naturaleza, las cuales sirven para que el ser humano salga del estado de guerra.

Locke (citado en Ávila y Gillezeau, 2009), es considerado el principal teórico del liberalismo. Este define el estado de la naturaleza como un estado de "paz, benevolencia y ayuda mutua". Pensaba que en el estado de la naturaleza el ser humano debería disfrutar de la libertad perfecta para manejar sus acciones y de tener la libre disponibilidad de sus propiedades y de su personal, claro está, dentro de la Ley Natural, pero sin pedir permiso y siendo independiente de la voluntad de otros individuos.

AGLALA ISNN 2215-7360

2014; 5 (1): 168-183 
Él se inclina por el pensamiento de la férrea subordinación de los poderes al poder legislativo y, éste a la vez, delegado a la voluntad popular, los cuales tienen el derecho de control sobre el uso del poder, para proteger la libertad de los excesos ilegales. Además, Locke le da a la ciudadanía una especie de derecho a la revolución, el cual debe de ejercerse en casos extremos cuando no exista otro medio de normalizar las cosas. De igual manera, Castillo (2014), expresa que Locke, en su obra: Dos tratado sobre el gobierno Civil de 1690, afirmó que la soberanía no está en el Estado sino en el pueblo, por lo que había el derecho a quitar al monarca cuando no cumpla su mandato que fue dado para que sirviera a la colectividad. Es más, le da al pueblo la facultad de modificar el sistema político por otro. Este personaje estuvo de acuerdo con la separación de la iglesia y el Estado.

Las reflexiones de Erasmo, (citado en Castillo, 2014), sobre el Estado y la sociedad, son opuestas al enfoque de Maquiavelo, dado que son los imperativos morales y religiosos los que definen las reglas de acción en la política. Además, considera que es mejor elegir al gobernante que investirlo por herencia y es adepto de una forma mixta de gobierno, en la cual se combinen los tres regímenes políticos: democracia, aristocracia y monarquía. Sin embargo, su principal preocupación no fue el tipo de gobierno sino el espíritu y el corazón de los gobernantes; es decir, su internalidad que les llevaba a ejecutar sus acciones.

Erasmo se enfoca en los ideales que busque la paz y da una crítica permanente a la guerra. Este, es denominado como el primer teorizador literario que busca la paz (Zweig, 1994). Lo cual permite inferir su énfasis en el respeto a la diversidad, el humanismo, el libre pensamiento y la justicia como ideal supremo de la raza humana.

Moro, (citado en Ávila y Gillezeau, 2009), en su humanismo cristiano, ofrece una visión del Estado radical al que ya existía. Este propone que el Estado sea igualitario por lo que no debe AGLALA ISNN 2215-7360

2014; 5 (1): 168-183 
de existir propiedad privada y que los magistrados deben ser elegidos por el pueblo. Esto se convierte en una utopía. Además, se opone a la explotación comercial y contenía ideas que reaparecen posteriormente en la teoría del socialismo.

Se puede inferir, que la Utopía de Moro, aunque contiene aspectos ilustrados y prácticos, es un ideal que se ha presentado en las sociedades comunitarias del pasado y, en algunos aspectos, es opuesta al avance de las fuerzas y estilos que permitirán la entrada a la modernidad, como son como la expansión del comercio y la separación de poderes, entre otras. Los humanistas de aquella época no prestaron mucha atención a los ideales políticas sino hasta fines del siglo XVI cuando hacen su aparición otras teorías que defienden a doctrina absolutista como la de John Bodín, entre otros.

Ávila y Gillezeau, (2009), expresan que Maquiavelo concentra su análisis en la teoría del poder y como mantenerlo, de manera independiente en sus medios para utilizarlo. Como el fin de Estado es garantizar la seguridad y el bienestar, el gobernante tiene derecho a valerse de cualquier medio para la consolidación y conservación del poder. Buscaba establecer la unidad italiana a modo de la República Romana clásica. Consideró necesario la figura del príncipe como autoridad. "A este personaje no le interesa cual es el mejor poder o el más legítimo, sino cuál es la técnica política más eficaz para conseguir y mantener el poder y el orden” (p. 156).

El pensamiento de Maquiavelo está sometido por el realismo político, el cual es una manera de expresarse del humanismo renacentista en la filosofía política y por los valores de su época y de la sociedad florentina.

Por otro lado, su pensamiento está orientado hacia las élites, considera al pueblo como una fuerza que puede ser manipulada. Su enfoque no era denunciar la anarquía feudal, sino establece fundamentos de la racionalidad en la política moderna.

AGLALA ISNN 2215-7360

2014; 5 (1): 168-183 
Otro personaje, Montesquieu, piensa que el poder es la ley de la razón humana porque gobierna todos los pueblos de la tierra; debido a ello, las leyes políticas y civiles de Estado no deben ser más que los asuntos particulares a los que se emplea la racionalidad humana.

Una nación está formado en base a diferencias, esto porque la diversidad de pueblos y costumbres hace que las leyes que convienen a una colectividad no la sea de la misma manera para otra. Además, para Montesquieu, la Constitución es un conglomerado de leyes fundamentales que organizan el poder de un Estado. Su principio es la libertad y el contrapeso entre poderes. La libertad es esencial, el cual es un bien que promueve el disfrute de los otros bienes. Esta libertad es establecida por la ley y quiere decir que un individuo no puede ser impelido a llevar a cabo una acción que las leyes no ordenan. La República es la forma de gobierno que puede ser democrática o aristocrática. El pueblo tiene un soberano poder. Además, un gobierno sin principios, aun las mejore leyes se convierten en malas. La fuerza de los principios lo implica todo.

En Bacon, el poder descansa en la Ciencia y esta es la base de transformación de la realidad. El buscaba establecer y extender el dominio de la humanidad sobre el universo, lo cual depende, tanto de las artes como de las ciencias. Es por ello que no se puede dominar la naturaleza sino se le obedece.

Expone que los descubrimientos ocupa el primer lugar entre las acciones humanas y beneficia más que cualquier reforma social o política. Ahora, solo se puede tomar control de la naturaleza conociendo su estructuración y respectivas leyes, la técnica no se puede llevar a cabo sin tener un conocimiento anterior de la realidad a través de la ciencia. Por lo tanto, los descubrimientos científicos y sus aplicaciones son de mucha utilidad porque abren puertas para nuevos hallazgos.

AGLALA ISNN 2215-7360

2014; 5 (1): 168-183 
Montalgne, piensa que la libertad es lo fundamental. Se obra se basa en la libertad y contra los tiranos. Se superponen y coinciden libertad religiosa y libertad políticas, opuestas a la intolerancia y a la tiranía. Estos son elementos esenciales en el poder y busca separar religión y Estado. Expone que se debe de humillar al hombre y su racionalidad, debido a que el ser humano no puede conseguir la verdad absoluta, sino relativa y así es como llega a conocerse a sí mismo y al mundo. En otras palabras, todo es relativo.

Montalgne centra su teoría en la libertad y con énfasis en la libertad individual, de creer y pensar. La libertad es así la razón de ser de todo. La libertad por lo demás, y especialmente, la libertad de pensamiento, constituye una de sus constantes, como materia, de la meditación filosófica y política. La guerra de religión es para este personaje un atentado sacrílego contra el misterio divino. Sólo Dios posee la verdad. Al hombre le corresponde únicamente buscarla sin cesar.

La doctrina de Rousseau fue esencial a la conformación de la nueva comunidad política que surge de la revolución y en la concepción de las repúblicas posteriores. Este efectúa una reformulación del sistema social, para hacer compatible la igualdad natural y el progreso tecnológico, convertido en degradación moral. Toda su filosofía está compenetrada en esta tesis. Rousseau será siempre el filósofo de los pobres, de los humillados en la Revolución Industrial.

A su vez, la libertad y la igualdad son inseparables. La ley es concebida como ser supremo, omnipotente y expresión de la voluntad general. Valoriza la educación de las virtudes cívicas, especialmente concebidas como amor a la patria, así como el reconocimiento del derecho indescriptible de la resistencia frente a la opresión, que posteriormente retoman múltiples constituciones republicanas.

AGLALA ISNN 2215-7360

2014; 5 (1): 168-183 
Para Rousseau, el hombre es bondadoso por naturaleza. Es el sistema social y específicamente la existencia del derecho de propiedad el que crea desigualdad y es inhumano. Es decir, que el hombre no ha sido influenciado por la civilización, es bueno y sociable. Reformula el sistema social, para hacer compatible la igualdad natural y el progreso tecnológico, convertido en degradación moral.

Voltaire (s/f), cree que la única fe es en la razón y en el progreso. No admite salvación y debido a ello es poco práctica cuando se quiere llevar su reflexión a la política o a la teología. Este personaje piensa que el primer deber del hombre es ser feliz y el primer deber de la filosofía es ayudarle a serlo. Nadie tiene nunca el deber de ser injusto, ni siquiera el Estado. Ve la desigualdad como natural, al expresar que todos son hombres, pero no miembros iguales de la sociedad. Además, piensa que el género humano tiene una impresionante y absurda fascinación por el mal.

En el aspecto económico, expresa que el comercio es la única fuente de progreso conocida y que potenciarlo es el deber obvio de cualquier gobernante ilustrado. El progreso es irreversible e imparable. Además, creía que el progreso material traería progreso moral.

Por último, Mirabeau, fue un defensor de la democracia y de la monarquía constitucional y estableció bases de los principios de la revolución. Defendió la implementación de una monarquía constitucional que estuviese limitada por una asamblea legislativa, tomando como ejemplo el modelo usado en la Gran Bretaña y en los escritos de Montequieu.

\section{Conclusiones}

La construcción del estado moderno significó para los países de Europa la transformación de una sociedad que había estado conformada por grupos feudales dispersados a lo largo de los AGLALA ISNN 2215-7360

2014; 5 (1): 168-183 
territorios, hacia una autoridad monárquica apoyada por los burgueses para concentrar el poder y establecerse como un estado absolutista. Los pensadores y filósofos de la época sustentan y justifican el autoritarismo por las premisas teóricas del Derecho Divino de los Reyes que lo plantea como el poder que proviene de Dios hacia el monarca y que es el individuo más capacitado para dirigir el futuro de sus súbditos.

Este cambio de pensamiento representó una nueva manera de pensar y de vivir, se va más allá del pesimismo y milenarismo medievales al optimismo del humanismo renacentista, lo que permitió un mayor crecimiento que se refleja en importantes descubrimientos, y el desarrollo de las artes, filosofía, arquitectura y ciencia. Por lo tanto, el nuevo paradigma permitió redefinir y proponer nuevos enfoques políticos, tales como: la soberanía sustentada en el pueblo, el control y la división de poderes en el estado, la separación de la iglesia con el estado, el pacifismo, el respeto a la libertad, la libertad de pensamiento y el estado igualitario.

\section{Referencias bibliográficas}

Avila, J. y Gillezeau, P. (2009). Poder, política, estado y democracia. ANIDIE - México. Universidad de Zulia - Venezuela.

Castillo, B. Poder y política en la era absolutista medieval del renacimiento. Revista Ciencias Sociales. 10, 3. Recuperado el 11 de Septiembre de 2014 de www.lamjol.info/index.php/FAREM/article/download/1613/1414

Echandi, M. (2007). El ejercicio del poder político en el renacimiento a través de El Príncipe.

Revista de Estudios, 20. Recuperado el 20 de mayo de 2014 de http://www.estudiosgenerales.ucr.ac.cr/estudios/no20/papers/iisec3.html

AGLALA ISNN 2215-7360

2014; 5 (1): 168-183 
Fazio, M. (2006). Historia de las ideas contemporáneas. Una lectura del proceso de secularización. España.

Fabal, G. (1973). Pensamiento social desde el medioevo hasta el siglo XIX. Cuba: Editorial de Ciencias Sociales del I.L.C.

Perry, A. (1987). El estado absolutista. Capítulo I. El estado absolutista en Occidente. México: Siglo XX editores.

Romano, R. y Tenenti, A. (1986). Los fundamentos del mundo moderno: edad media tardía, reforma y renacimiento. Historia Universal Siglo XXI. España: Siiglo XXI Editores S.A. Voltaire. (s/f). Cartas Filosóficas. Recuperado el 10 de noviembre de 2014 de http://www.bsolot.info/wp-content/uploads/2011/02/Voltaire-Cartas_filosoficas.pdf

Zweig, S. (1944). Erasmo de Rotterdam, triunfo y tragedia. Recuperado el 25 de mayo de http://www.remq.edu.ec/libros/Stefan\%20Zweig\%20-

\%20Erasmo\%20de\%20Rotterdam,\%20Triunfo\%20y\%20Tragedia.pdf

AGLALA ISNN 2215-7360

2014; 5 (1): 168-183 